\title{
The Effects of the Mixed Sensory-motor-perception Training on Fill in the Blanks Performances of the Students with Learning Disorder
}

\author{
Raheleh Haghighatzade $^{1}$, Shole Amiri ${ }^{1} \&$ Hossein Molavi ${ }^{1}$ \\ ${ }^{1}$ Department of Psychology, Faculty of Isfahan University, Isfahan, Iran \\ Correspondence: Raheleh Haghighatzade, Department of Psychology, Faculty of Isfahan University, Isfahan, \\ 8174673441, Iran. E-mail: r.haghighat.edu@gmail.com
}

Received: February 16, 2012

Accepted: February 29, $2012 \quad$ Published: June 1, 2012

doi:10.5539/ijps.v4n2p169

URL: http://dx.doi.org/10.5539/ijps.v4n2p169

\begin{abstract}
The aim of the present study is to survey the effects of the mixed sensory -motor-perception training on the fill in the blanks performances of the Students whith with reading disorder in Isfahan. The statistical population includes 30 dyslexia dyslexic students from the learning disorder center in Isfahan. The samples are selected randomly. They and included 30 dyslexia thirty dyslexic students at the learning disorder center of Isfahan, who are were divided in two fifteen individual groups. One of them selected as an experimental group, and the other one as the control group.

The research instruments are were: Demographic tests, Wechsler Intelligence Scale for children, and the reading screening tests which are were used as the pre-test and the post-test. While the experimental group was individually exposed to ten one-hour sessions of mixed-sensory-motor-perception training, the control group underwent no special training and interferences. The results obtained by the statistical method of covariance analysis revealed that there was a significant difference $(p<0.001)$ between the average scores of the post-tests of the experimental and the control group. The overall results of the present study showed that the mixed-sensory-motor-perception training has had been effective on the dyslexia dyslexic students' fill in the blanks performances and has had made a significant raise in the average scores of their post-tests.
\end{abstract}

Keywords: mixed-sensory-motor-perception t- training, fill in the blanks performances

\section{Introduction}

Reading disorder is one of the commonest learning disorders overwhelming almost $4 \%$ of the students. Reading disorder will be specified by the defects in the ability to recognize the terms, reading slowly, a weak perception in the absence of intelligence, or a significant physical dysfunction. These children have difficulty in distinguishing the letters which are different from each other only in terms of their sizes and forms, especially the ones which differ only in terms of their special directions or the lengths of the lines. Also they have difficulty analyzing the words and dividing them to various syllables (Studly, Stain, and Harrison, 2006). The topic of sense, perception, and motor has found a special status in the evaluation of most of the disorders, such as, dyslexia. Reading, undoubtedly is one of the most important and complex training activities of the students at early school years. Reading means receiving ideas, experiences, sensations, and concepts. In fact, it's an activity which allows an individual to obtain a great knowledge about the present world (Hassanloo, and Pushne, 1996). Most of the students with difficulty in learning face some problems in visual or audio perceptions. They may lack ability to write words or to realize the geometrical forms. Some have a weak memory, or reveal hyperactivity, which all together indicate the hidden disorders in the brain. These disorders may affect the other sensory ways as well, including the auditory or touching senses which lead to learning disorders. As an example, the children with dyslexia have difficulty realizing the successive touching stimuli quickly. For instance, if you ask them to put their hand under something and touch one of their fingers, they can say which finger they have touched, while if you ask them to touch two fingers quickly, one after another, they recognize only one of the fingers (Zarbakhsh, 1999). The most significant evidences about reading disorders turn back to malfunctions of the auditory, verbal, or visual senses. This field includes a great literature, such as, the clinical and experimental studies of the children with reading and learning problems.

Also the studies on 200 students with reading difficulties represented that based on the standard tests, $90 \%$ of them had a verbal defect, and 96\% were with verbal perception and recognition problems (Bardford, 2005). A 
research on multi-sensory methods applied for the students with reading disorders was done by Auto and Mack (1993), and the results indicated that this method has been very effective and has been confirmed in the empirical research.

In 1993, finally Bangur concluded that the sensory-perception and motor-perception malfunctions lead to a fault in the speaking ability of the students with reading problems (Nickleson and Facket, 1999). A child with learning difficulty faces so many problems and disorders not only in learning, but also in all other aspects, such as, a sensory perception, motor and even social aspects. Based on previous research, all old methods for treating learning disorders only focused on the improvement and the growth of one aspect of an individual's growth. In this research, all the sensory perception and motor methods were mixed. The present researcher decided to mix all the sensory perception and motor methods of the children's growth field in order to find a proper method to improve a child with learning disorders. Considering the previous studies, they found no similar research in this field and this itself can be a reason for the innovation of the present subject. On the other hand, compared to other learning disabilities, dyslexia takes a higher percent, as most of the students with fill in the blanks disabilities have difficulties in expressing letters or analyzing the words. A lot of other studies are done in this respect, realizing the phonology as one of the basic problems of the students with reading disorders (Servic \& Visroo, 2001, Ramoos, 2005, Visoor 2004). As a result, the present study tries to measure the effects of sensory-perception-motor training on the above dyslexic children cases.

\section{Method}

\subsection{The Participants and the Research Design}

This is an empirical study with pre-tests and post-tests, as well as, the control group. The pre-tests are considered for each group and then an independent variable was applied for the experimental group, while the control group received no training, and finally both groups underwent a post-test to compare their scores. The effectiveness rate of the training method was evaluated. In this research, some disturbing variables were used by the random method for controlling the variables. The cases under study were selected from among the students from the schools in various areas of Isfahan city. The cases were referred to the learning disorder consulting center in Isfahan by the teachers working at the educational and training system in Isfahan.

30 girls at the third grade, confirmed by their teachers as the students with learning difficulty were selected as the cases, then the intelligence test and the Wechsler form was performed for them in order to evaluate and determine if their intelligence level was normal. Once again, two groups, each one including 15 individuals in the control and the experimental groups were selected.

\section{Research and Instruments:}

In the present study, the intelligence and the Wechsler tests were applied in order to ensure the absence of the intelligence defects. The Wechsler intelligence scale for the children (WISC-R) was provided by David Wechsler for the assessment of the children intelligence assessment. This scale was reconsidered in 1974 and after determining the disorders; it changed into the new scale of Wechsler for the children. In order to determine the validity of the scale, the validity of the reassessment of the tests and IQ tests and the validity variances of the tests of the scales were studied again. In the reassessment validity, the co-efficiencies of the test validity differed between $44 \%$ and $94 \%$ (While the medium of each validity co-efficiency being $73 \%$ ). In descriptive validity, the validity co-efficiencies of the tests varied from $42 \%$ to $90 \%$ (the medium of the validity co-efficiency being $69 \%$ ). Comparing the simultaneous validity of this scale with Wipsy validity, we find that the covariance co-efficiency of the verbal and practical IQ tests, and the total amount for the two scales were $84 \%, 74 \%$ and $85 \%$ in respect.

\subsection{Reading Test}

This test was designed to provide some information about the special skills of reading and it evaluates the skills which are directly related to the training in the classroom. This test is provided based on "equal" evaluation form (1977) which is validated by Badiean (1375). The validity of this test for the third grade students was considered as $92 \%$, and it involves the commonest problems included in the reading schedules of the students. This test involves 8 sub-tests. The score of the tests are considered for the tests 1 to 6 , while the score of 20 was considered for the sub-tests of 7 and 8 , and the total score is considered as 100 . One score is decreased for each error; therefore, considering the cut score of 48 for each test, any student getting the score of 48 or less will be evaluated as a dyslexia student.

\subsection{Population Questionnaire}

This questionnaire involves some questions about the number of the people in each family, the parents' 
occupation, and their educational levels, their economical status, and their age.

\section{Method and Data Collecting:}

This design and the mixed program include a ten one-hour session treatment, and the explanation of them in details is stated in the following.

\section{The First to Fifth Session:}

Aim: Increasing the visual and the motor conception skills, increasing the speed and reaction in finding directions, and increasing the short-term memory, the comprehension skills in understanding several successive sentences.

\section{Programs:}

To increase the visual- motor skills, and to coordinate eyes and hands, the FRASING test and the related exercises are used which resulted in the visual comprehension growth

\section{The Eye Following Exercises:}

The students would follow an object in all directions, up, down, right, and left without moving their heads.

\section{Increasing the reaction speed in finding directions:}

Several big arrows are drawn on various directions on a piece of paper and the students are asked to tell the direction of each arrow. After several sessions, the size of the arrows will be decreased and they will be closer together.

Increasing the short-term memory: (Straight, vice versa)

For this exercise, the short-term memory tests and Wechsler numbers are applied.

The ability to increase the auditory comprehension of two following sentences:

Here the students are asked to do an order including more than one sentence. Little by little, the number of the sentences will be increased.

\section{The Sixth to Ten sessions:}

Purpose: Strengthening the big movements and the balance, recognizing the letters, increasing the sensory perception, increasing the special perceptions.

\section{Strengthening the big movements and balances:}

Some exercises, such as, walking on the edges of the blocks, standing on one leg, and playing GrdooShekastyam game, walking backward, throwing something backward, and catching the ball. The child must dance at home for 15 minutes.

\section{Strengthening the power and the visual distinction:}

To empower the visual distinction ability in realizing the letters of each word and recognizing the letters used in a sentence in a way that several words are written on a piece of paper and the students are asked to find some special letters in the words and draw a red line around them.

\section{Lip reading:}

The aim of this exercise is increasing the precision and the concentration of the students in looking around. The trainer sits face to face with the child and moves his lips the same way he wants to say a word, but he doesn't utter any sound. The students should realize the words by the movement of the mouth and the lips.

\section{Increasing the sensory perceptions:}

\section{Playing with sand:}

Some tools, such as, a cube, a spoon, or a ball are hidden in sand and the students are asked to find these tools. Finding the intended tools, they should guess the object only through touching, not looking at what they have in their hands.

\section{Results}

As observed in table 1 , the difference between the mean of the balanced scores and the reading scales in both control and the experimental groups is significant $(\mathrm{p}=0.0001)$. 
Table 1. The results of the covariance analysis of the effect of membership on the fill in the blanks performances of the control, and the experimental groups

\begin{tabular}{llllllll}
\hline Source & $\begin{array}{l}\text { Sum of } \\
\text { squares }\end{array}$ & $\begin{array}{l}\text { Mean } \\
\text { square }\end{array}$ & F & Sig. & Eta & $\begin{array}{l}\text { Observed } \\
\text { power }\end{array}$ \\
\hline pre & $62 / 36$ & 1 & $62 / 36$ & $44 / 27$ & $0 / 0001$ & 45 & 1 \\
Group & $25 / 11$ & 1 & $25 / 11$ & $11 / 06$ & $0 / 0001$ & 50 & $0 / 90$ \\
\hline
\end{tabular}

The results of the test also reveal the non-parametric Man Wittny, which indicate that the difference between the scores of the two groups is significant.

As a result, this hypothesis is confirmed. The effect size equals to $50 \%$, meaning that $50 \%$ of the score variance of the students' performances relates to group memberships. The statistical power of $90 \%$ shows that the sample volume for this hypothesis has been sufficient.

Table 2. The averages and the standard deviations of the post-tests in the experimental and the control groups

\begin{tabular}{lcll}
\hline \multirow{2}{*}{ Group } & $\mathrm{N}$ & \multicolumn{2}{c}{ Post test } \\
\cline { 3 - 4 } & 15 & $4 / 85$ & $1 / 6$ \\
\hline Experiment & $\begin{array}{l}\text { Std. } \\
\text { deviation }\end{array}$ \\
Control & 15 & 3 & $2 / 4$ \\
\hline
\end{tabular}

Based on the data of the table 2, the average of the post-test scores in the experimental group is $4 / 85$, and the average scores in the control group is 3 , and as represented in this table, there is a significant difference between the reading scores of the post-tests of the two groups.

The comparison of the average scores of the reading post-tests of these groups are indicated in figure1.

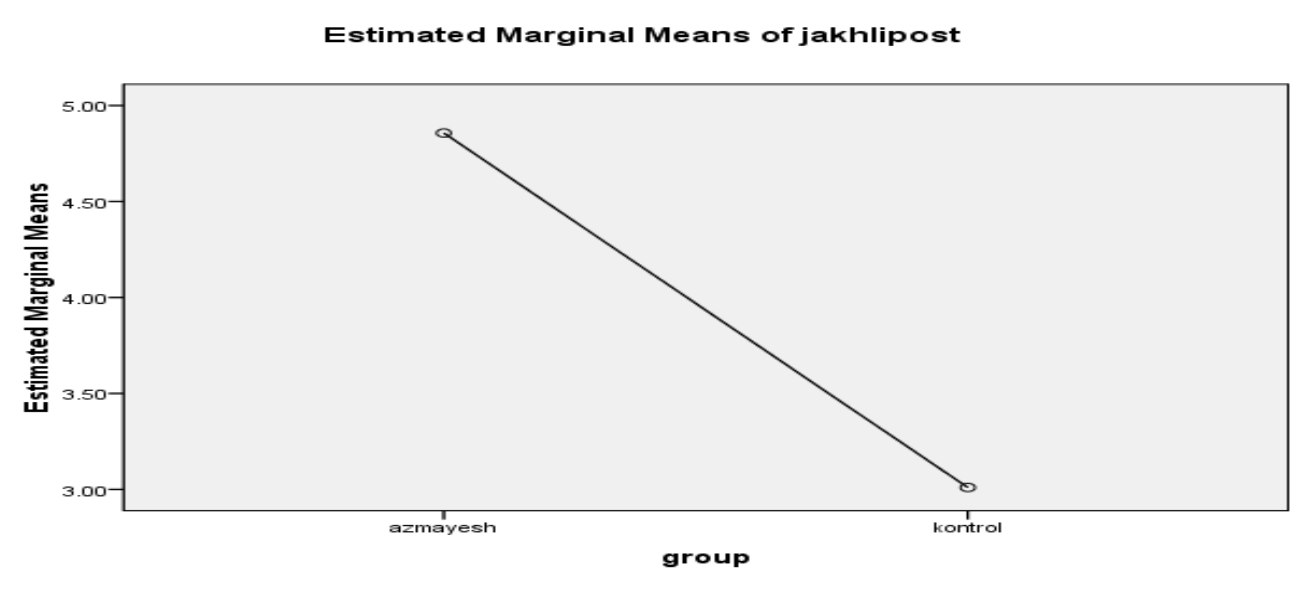

Figure 1. Estimated marginal means of fill in the blanks performances

\section{Discussion}

The children are absorbed more slowly than their peers and environmental stimuli to learn the same skills later Perception system for people with learning disability, has suffered only acts differently from ordinary people. This assignment (fill in the blank) in addition to basic ability to such as precision, focus and attention needed is the ability to understand high. Children can read the text, it makes sense. And with the general subject and text information that is gained can guess. What word in the blank with text that can be. Most children with learning 
disorders have been Deficienciess in visual perception and reading comprehension. Are not able to get the meaning of the text reads Visual stimulus was the same as the text and fill in the blank test. The exercise was performed in ten sessions, the training was to increase comprehension and attention (like Storytelling and select a theme for the story). So unexpected was this exercise that increases students' scores on the subtests.

The results of this hypothesis coordinates with the results obtained in the research by karati (1999), Kakaee (1382), Yaghobi (1384), Zeini vand (1386), Asadi dost (1386), Rabinson (2000).

\section{References}

Bardford J. (2005). Using multi sensory Teaching Methods. Retrieved from http:// www. dyslexia .parent. com Cratty, A. (1999). Motor aspect of dyslexia. London: Rutledge .

Hassanloo, Tahere., \& Poshne, Kambiz. (1385). A Survey of the effects of the mixed-motor-perception on Reading Performances of Dyslexia Students of the third and fourth grade in Zanjan city. Special education and Training, 61, 3-11.

Nicolson, R. I., \& Fawcett, A. J. (1999). Developmental dyslexia: The role of the cerebellum. Netherland: Klumwer Academic publishers.

Robbinson Melvin P. (2006). A study of the validity of Delacato's Theory of Neurolocical Organazition. Exeptional Children, 32, 517-533.

Servic, E., \& Virsu, V. (2001). Temporal order and processing acuity of visual, auditory and tactile perception in developmentally dyslexic young adults. Cognitive, Affective and Behavioral Neuroscience, 12, 349-410.

Shahim, Sima. (1383). A Revised Comparison of Weksler Intelligence for Children, Matching and finding orders. Shiraz: Shiraz University Publication.

Stoodly, J. S, Harrison, P. D. E., \& Stein. F. J. (2006). Implict motor learning deficitis in dyslexic adults. Neuro psychologia, 44 (5), 795-798. http://dx.doi.org/10.1016/j.neuropsychologia.2005.07.009

Yaghobi, Abolghasem. (1384). The Influence of Training Methods On improving the Reading Function of the DYSLEXIA Students. Phd. Thesis, Alame University, Psychology Faculty.

Zarbakhsh, Mohammadreza. (1378). A survey of the multisensory method of Orton on reading disorders in the dyslexia students of the second and third grade. M. A thesis of Public Psychology, Isfahan University, Isfahan University (not published yet). 